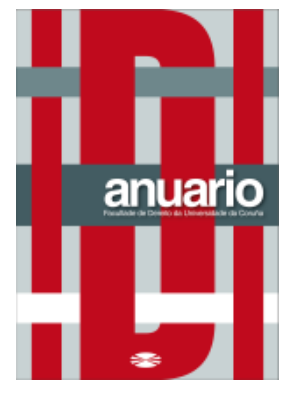

Anuario da Facultade de Dereito da Universidade da Coruña

Vol. 22 (2018), pp. 176-187

ISSNe: 2530-6324 || ISSN: 1138-039X

DOI: https://doi.org/10.17979/afdudc.2018.22.0.5181

\title{
NUEVOS DERECHOS \\ EN LA COLABORACIÓN PÚBLICO-PRIVADA
}

\author{
ANTONIO MANIATIS \\ Ex Docente de la Universidad de Castilla - La Mancha
}

\begin{abstract}
Resumen: En el marco de la colaboración público-privada (CPP) existe una regulación que no asegura la selección de la mejora empresa como supervisora, dado que el comité competente se limita a examinar si el propuesto supervisor cumple los criterios previstos, en base de un procedimiento llamado «on-off». En todo caso, el contratista tiene los nuevos derechos a seleccionar a su supervisor y a ofrecerle una remuneración suplementaria. De sobra, la CPP, emergida en la época corriente de los derechos fundamentales de cuarta generación, es asociada al derecho a la serenidad, el cual resulta de la directiva 2014/61/UE en materia de coordinación de obras civiles, y por lo general es promotora de los nuevos derechos, de cuarta generación, o de cuasi nuevos derechos, ejemplificados por el derecho al medio ambiente.
\end{abstract}

Palabras clave: colaboración público-privada (CPP); derecho a la serenidad; derecho al medio ambiente; derecho a ofrecer una remuneración suplementaria al supervisor; derecho a seleccionar al supervisor; nuevos derechos

Abstract: In the framework of the public-private partnership (PPP) there is a regulation that does not ensure the selection of the best supervising enterprise, given that the competent committee is limited to examining whether the supervisor meets the established criteria or not, on the basis of a procedure called "on-off". Anyway, the contractor has the new rights to select his supervisor and to offer him a supplementary reward. Besides, PPP, which emerged in the current era of $4 \mathrm{G}$ fundamental rights, is associated to the right to serenity, which results from directive 2014/61/EU in the matter of coordination of civil works, and in general is conducive 
to new rights, namely $4 \mathrm{G}$ rights, or to quasi new ones, exemplified by the right to the environment.

Keywords: Public - Private Partnership (PPP); right to serenity; right to the environment; right to offer a supplementary reward to the supervisor; right to select the supervisor; new rights

SUMARIO: I. INTRODUCCIÓN. II. LA PARTICIPACIÓN A LA SOCIEDAD DE LA INFORMACIÓN Y LOS DERECHOS DEL CONTRATISTA EN EL ORDENAMIENTO GRIEGO. III. LA CPP COMO PROMOTORA DE NUEVOS DERECHOS Y DE CUASI NUEVOS DERECHOS. IV. LA CUESTIÓN DEL DERECHO HIPOTÉTICO A LA SERENIDAD. V. A MODO DE CONCLUSIONES

\section{INTRODUCCIÓN}

La colaboración público-privada (CPP) constituye una especie de cooperación institucionalizada entre los dos sectores de la economía, por lo que se refiere a las obras técnicas o la prestación de servicios. Estos proyectos se denominan en inglés «Public - Private Partnerships (PPP o P3)» ${ }^{1}$. Se trata de una asociación de cooperación con base a la experiencia («savoir-faire») de cada asociado, la cual responde mejor a las necesidades públicas, a través de una repartición adecuada de los necesarios recursos y de los riesgos.

La CPP se introdujo por primera vez en el Reino Unido, en 1992, en forma del programa 'Private Finance Initiative'. Esta forma de contratación incluía objetos no recíprocos, como escuelas, cárceles y hospitales, para que el poder estatal evite el coste político implicado por la institucionalización de cargas recíprocas. Contrariamente al modelo tradicional similar de concesión, destinado a objetos recíprocos, esta innovación era relativa a un privilegio del sector privado, dado que su remuneración estaba garantizada por aquel público. Habitualmente, la CPP está dedicada a los objetos no recíprocos a nivel internacional, sin que exista una norma jurídica $^{2}$. Resulta una complementariedad informal de este modelo frente a los contratos de concesión, con los cuales hace parte de la misma metodología diacrónica. En todo caso, ambos tipos de contratación están sujetos a la regla (non-jurídica) de $50 \%$, adoptada por Eurostat y el Banco Europeo de Inversiones (BEI) ${ }^{3}$.

En España, la colaboración entre el sector público y aquel privado era muy usada sin un apoyo jurídico (por ejemplo, el programa del Ministerio de Medio Ambiente, de 2004 «A.g.u.a» Actuaciones para la Gestión y Utilización del Agua), por los últimos

\footnotetext{
1 RAMÍREZ MARTÍNEZ, S., «Ley de Asociaciones Público-Privadas», Energía a debate, 2012.

${ }^{2}$ MANIATIS, A., «The concession nature of Public - Private Partnership», e-JST, 11 (3), 2016, p. 29.

${ }^{3}$ MANIATIS, A., EVANGELATOU K., MANOLIADIS, O., «An assessment of the French Public Private Partnership Law», e-JST, 11 (4), 2016, p. 13.
} 
años antes de adoptar un cuadro institucional ${ }^{4}$. La ley «sobre los contratos de la administración pública»30/2007, del 31/10/2007, incorporó de manera decidida el cambio de perspectiva que desde hace años había impuesto en este sector el Derecho Comunitario y que sin embargo la legislación española se resistía a asumir plenamente $^{5}$. La nueva ley de contratación pública, recientemente introducida en el ordenamiento de España, elimina la figura del contrato de CPP, pero sin que el derecho de la Unión europea excluya la posibilidad de hacer uso de este tipo de contratos. Entonces, este hecho no podría deslegitimar una investigación sobre la CPP, la cual ha sido un modelo internacional de contratación. Sería interesante analizar este modelo en materia de los nuevos derechos, particularmente del contratista y de los usuarios. Ciertamente, el fenómeno de la contratación pública y de la CPP es estrechamente asociado a los derechos fundamentales de las últimas generaciones de derechos, ejemplificados por el derecho al agua ${ }^{6}$. Esta garantía debería consagrarse de manera explícita y simple, exenta de prejuicios y distorsiones ${ }^{7}$. El presente análisis va a focalizar en varios nuevos derechos, en principio de las empresas privadas, en el marco de la CPP.

\section{LA PARTICIPACIÓN A LA SOCIEDAD DE LA INFORMACIÓN Y LOS DERECHOS DEL CONTRATISTA EN EL ORDENAMIENTO GRIEGO}

En la Constitución griega se ha incorporado una nueva disposición, el artículo 5A par. 2a, a través de la revisión de 2001. En esta disposición se prevé que «Cada persona tiene el derecho a la participación a la Sociedad de la Información».

Algunos años después, la ley 3389/2005 introdujo formalmente el modelo de la CPP por primera vez en el ordenamiento griego. Según el artículo 17, cada contrato debe citar el modo de ejercicio de la supervisión de la ejecución y operación de la obra y de la prestación del servicio, sea a través de compañías independientes optadas por las partes contratantes en común sea mediante los servicios competentes del sector público. La ley implícita la selección del supervisor por las dos partes en común. Sin embargo, la practica ha designado una aplicación distinta de ese modelo, según la experiencia europea; cada candidato para el puesto del contratista debe mencionar, durante la licitación, el supervisor propuesto. Por un lado, las empresas que desempeñan el papel del supervisor son compañías habitualmente no griegas, de alto nivel. Resulta lógico que éstas no arriesgarían a comprometer su fama para favorecer al contratista.

Por otro lado, no es clara la razón de encargar una empresa privada «independiente» para hacer lo que en todo caso hace, incluso informalmente, el servicio técnico del sector público. Ésta es la diferencia esencial entre una obra técnica pública y alguna

\footnotetext{
${ }^{4}$ MORENO MOLINA, J. A., PLEITE GUADAMILlAS, F., La nueva ley de Contratos del Sector Público. Estudio sistemático, La Ley, Madrid 2008, p. 913.

5 MORENO MOLINA, J. A. «El ámbito objetivo de aplicación de la ley de contratos del sector público», Documentación Administrativa núm. 274-275, 2006, p. 47.

${ }^{6}$ MANiATIS, A., El Derecho al Agua y la Contratación Pública. Estudio de Derecho Público, Editorial Académica Española, 2018.

${ }^{7}$ MANIATIS, A., «Derecho hidráulico y contratación pública», Observatorio Contratación Pública, 05.03.2018.
} 
privada; el maestro de la obra tiene su propio servicio técnico y puede administrar y supervisar la ejecución de la obra mientras el propietario de un proyecto privado normalmente es ajeno al contexto de las construcciones. Dado que los contratistas cuentan con una oficina de calidad de la producción, el supervisor parece a una oficina externa de calidad que actúa por cuenta del supervisado.

Estas remarcas valen por lo que se refiere a los contratos del tipo «BOT, Build, Operate and Transfer», que la empresa pública «Sociedad de la Información S.A.» ha estipulado. Se trata del método más utilizado en materia de infraestructuras a dimensiones grandes, como puentes, carreteras, puertos, instalaciones energéticas etc. en el cuadro de la tipología internacional de la $\mathrm{CPP}^{8}$.

La empresa precitada ha focalizado en la prestación de servicios de banda ancha al por mayor, a los prestadores de las telecomunicaciones a través de la valorización de la red que va a desarrollarse en el cuadro de la obra. Están creadas infraestructuras en las regiones «blancas» del entero territorio de Grecia. Tres contratos parecidos ejemplifican el modelo de la CPP, el cual es en tal caso de naturaleza parcialmente recíproca, dado que a causa de las condiciones que rigen a las regiones - objetivo, un investimento de tal tipo no podría ser plenamente recíproco y rentable en su conjunto. Por esa razón, existe una contribución de la financiación de parte de la autoridad de adjudicación por la Fase A («Build», es decir de construcción), de duración de 24 meses, y no por la Fase B («Operate», relativa a la función de la operación), de duración de 180 meses.

Cada candidato era obligado a seleccionar una compañía especializada en materia de las infraestructuras de banda ancha, la cual sería encargada de hacer el control cualitativo y cuantitativo de la materialización de la obra, antes de cada solicitud de pago del contratista, que no es ajeno a ese procedimiento. Ello ha incluido este coste en su oferta y debe ser cubierto en términos de seguro de responsabilidad profesional.

Por lo que se refiere a la relación entre el contratista y el supervisor, no está asegurada la selección de la mejora empresa como supervisora, dado que el comité competente se limita a examinar si el propuesto supervisor cumple los criterios previstos, en base de un procedimiento llamado «on-off». Si esos no están cumplidos, la oferta del candidato correspondiente para la CPP está anulada. Si un candidato propone una empresa que es mejora a las otras propuestas, no adquiere una ventaja, pues carece de motivación. De sobra, por la fase A el contratista a través de su propia participación facultativa está legitimado a aumentar la remuneración del supervisor, como un empleador que ofrece un bono a su empleado. Al contrario, el coste de la remuneración por la Fase B está cubierto únicamente a través de una financiación por la parte contratante privada.

Además, dado que los gestores de las redes que van a crearse se convierten en prestadores de redes de servicios electrónicos, están sujetos al régimen legislativo de obligaciones, vigente en materia de este mercado. Se trata de la ley 4010/2012, según la cual la «Comisión Nacional de Telecomunicaciones y Correos» tiene una competencia de control, de supervisión y de imposición de sanciones. Esta autoridad (administrativa) independiente, la cual no tiene una consagración constitucional

\footnotetext{
${ }^{8}$ FABI, F., LOIERO, R., PROFITI, F. S. E., Il Partenariato Pubblico - Privato nell'ordinamento giuridico nazionale, comunitario ed internazionale, Dike Giuridica Editrice, 2015, p. 73.
} 
contrariamente a tres otras autoridades independientes similares, es competente para realizar auditorías de costes del sistema correspondiente del contratista. Sin embargo, el control no se realiza por la autoridad misma sino por un auditor independiente especializado.

El auditor, el cual puede ser diferente del supervisor ya mencionado, desempeña un papel muy crucial por la Fase B (no también por la Fase A) de la obra porque ofrece su ayuda a la autoridad independiente en el cumplimiento de sus obligaciones. Está seleccionado por la Comisión precitada, mediante un procedimiento definido y ejercido por ella. Su financiación está realizada solamente por el contratista, dado que en todo caso la financiación de parte del Estado es ajena a la Fase B. El procedimiento de selección es ventajoso en términos del principio de transparencia porque el pago de la remuneración del auditor está fijado de un modo predefinido en la declaración de la licitación. En todo caso, no existe una suficiente razón de interés público para la externalización de los servicios de control de parte del Estado, sin hablar de la particularidad de la novedad institucional que consiste en una autoridad administrativa independiente especializada.

\section{LA CPP COMO PROMOTORA DE NUEVOS DERECHOS Y DE CUASI NUEVOS DERECHOS}

La CPP constituye una innovación en el marco de la contratación pública y por eso es intrínsecamente favorable a nuevos proyectos del sector público, muchas veces asociados a derechos originales en el derecho comparado. Ha promocionado, más o menos, varios nuevos derechos, es decir de cuarta generación en la historia de consagración de los derechos fundamentales. Esta última generación es relativa sobre todo a las tecnologías, particularmente de información y comunicación, y a la cuestión de mitigación del cambio climático. El primer tratado internacional en la materia se concluyó en 1992 y entró en vigor el 21 de marzo de 1994. Se trata de la Convención Marco de las Naciones Unidas sobre el Cambio Climático. Después de ese texto, sigue una serie de conferencias de las partes contratantes, conocidas como 'Conference of Parties (COP)', En 1997, de la 'COP 3'" resultó el Protocolo de Kioto, el cual completa el contenido de la Convención, fijando algunos objetivos vinculantes, como reducir las emisiones de seis gases de efecto invernadero que causan el calentamiento global. En el protocolo se acordó una reducción de al menos un 5\% de las emisiones de estos gases por el período 2008-2012 frente a las emisiones de 1990. Entonces, la era de la cuarta generación, inaugurada por la adopción de la convención inicial sobre el cambio climático, coincide con la época del mecanismo de CPP.

Además, no sólo derechos de cuarta generación sino también cuasi nuevos derechos podrían promocionarse mediante la metodología de CPP. Éste es el caso de los derechos del sector turístico, consistentes en los derechos humanos al turismo y a la hospitalidad $^{9}$. Estos derechos fueron incorporados por primera vez en 1948 en la Constitución de Italia sin el uso del término "derechos" mientras la Constitución

${ }^{9}$ VAINOPOULOS, R., MERCIER, S., Le Tourisme, Le Cavalier Bleu Éditions, 2009. 
española de 1978 imitó el texto italiano. Desde 2001, mediante una revisión del artículo 117 que hacía referencia al turismo y a la industria de los hoteles, estos bienes han perdido su estatuto constitucional, en el ordenamiento arquetípico de Italia. En la época actual una garantía constitucional explícita, en forma sea de derecho sea de garantía institucional, es cuasi inexistente en las Constituciones, con la importante excepción del texto venezolano de 1999. El turismo debería fortalecerse directamente a través de la CPP, por ejemplo el Estado griego ha decidido la construcción de una marina en su ex capital, la ciudad de Nauplia. En todo caso, el turismo puede apoyarse por la contratación relativa al sector de energía, como es el caso reciente de intervención energética consistente en la gestión de la basura, en una destinación emblemática como Mallorca.

Resulta que la CPP es asociada al derecho genérico a la energía, el cual constituye un cuasi nuevo derecho, es decir de tercera generación. Evidentemente, la era actual de cuarta generación incluye el derecho especial a la eficiencia energética, sobre todo de los edificios. Según una aproximación similar de la doctrina, existe la siguiente representación piramidal del contenido del derecho humano a la energía, cuya base constituye el nivel mínimo y cuya cúspide el nivel máximo ${ }^{10}$ :

El derecho a la energía como opción de vida (p. ej. Vivir con o sin energía eléctrica) se colocaría en el primer escalón.

Se situarían en el segundo escalón aquellos elementos jurídicos que permiten gozar de energía cada vez que el individuo haya decidido vivir con ella (principio de universalidad).

En el tercer nivel están las iniciativas político-jurídicas que tienden a garantizar la prestación del servicio a quienes no puedan asumir los costes correspondientes. Así, frente a la posición activa de los titulares del derecho a la energía, existe la posición pasiva de los poderes públicos que deberían establecer los mecanismos apropiados para garantizar el servicio a toda la población (por ejemplo, la técnica de los valeenergía).

En el último escalón se colocaría la opción pro-ambiente, consistente en la facultad para el titular del derecho a la energía de elegir entre fuentes renovables y convencionales.

Además, cabe señalar que determinadas elecciones políticas pueden influir sobre la disponibilidad de un recurso energético: considérese el caso de la energía atómica en Italia $^{11}$. La CPP, al menos en el ordenamiento francés, no ha sido asociada al uso de un tipo ambivalente de energía como la energía nuclear, pese al hecho de que recientemente existió un proyecto en la materia. En todo caso, el derecho de la Unión europea no impone un modo determinado de gestión de los servicios públicos, dejando a los Estados miembros, por aplicación del principio de subsidiariedad que

\footnotetext{
${ }^{10}$ PARENTE, A., Principios de derecho europeo de energía, Aranzadi Thomson Reuters 2010, págs. 296-297.

${ }^{11}$ PARENTE, A., Principios de derecho europeo de energía, Aranzadi Thomson Reuters 2010, págs. 56-57.
} 
escojan el modo de gestión que estimen más conveniente ${ }^{12}$. Este pluralismo potencial es obvio en el sector de suministro de agua, por ejemplo en Francia y en España.

Es también notable que en Francia, antes de la adopción del cuadro legislativo sobre la CPP, habían sido introducidas delegaciones de servicio público y concesiones, como en materia del museo Jacquemart - André. El Instituto de Francia fue el primero, en 1990, en realizar delegaciones de servicio a un empresario privado («Cultures espaces»). El museo Jacquemart - André, en Paris, y las villas Kérylos y Ephrussi en la provincia, han sucesivamente constituido objeto de una transmisión de la gestión. En 1995 el museo se limitaba en un número de 20.000 visitantes anualmente y tenía un déficit operacional de 459.000 euros. Desde el inicio de la intervención del empresario privado, tuvo lugar un aumento del número de visitantes (entre 180.000 y 240.000) y también de los ingresos del Instituto, que oscilaron entre 137.000 y 150.000 euros. No se trata de un abandono de responsabilidades de parte del sector público sino de una repartición de competencias. El Estado prefiere concentrarse en la colección y la rehabilitación de las obras ${ }^{13}$. Este ejemplo se considera como de mayor importancia, dado que resultó que un objeto contractual, que por su propia definición no es lucrativo, es compatible con el modelo de concesión, tradicionalmente destinado a los objetos recíprocos, como ya señalado.

Basado en ese precedente, el modelo de CPP, que tiene como órgano de competencia decisiva la «MAPPP» (Misión de Apoyo en la realización de la contratación de colaboración público-privada), es decir un organismo tecnocrático y no político, es utilizado en el sector cultural, con varios casos de éxito ${ }^{14}$. Éste es el caso del Museo nacional de las culturas de Europa y Mediterráneo, con sede en Marsella. Esta obra se puso en operación en 2013, año en el cual el mayor puerto del Mediterráneo era la capital cultural de Europa. La intervención en el puerto tenía un gran éxito, incluyendo la operación de una torre cerrada desde hace siglos, la fortaleza de San Juan $^{15}$.

Además, en 2007 el gobierno querría iniciar su aproximación a la CPP en el sector cultural a través de la modernización del museo en Versalles. Los jardines de esta destinación turística de índole ecuménico hacen parte del patrimonio natural de la humanidad, en virtud de la Convención sobre la protección del patrimonio mundial, cultural y natural, de 1972. El contenido de esta Convención ejemplifica los derechos fundamentales de tercera generación, la cual comenzó en el mismo año, a través de este texto y sobre todo la declaración de Estocolmo sobre el medio ambiente humano.

La ejecución del contrato relativo al sistema de la emisión de los billetes del museo se interrumpió definitivamente dado que emergieron serios problemas, en detrimento de

\footnotetext{
12 TORNOS MAS, J. (Coord.), El servicio de suministro de agua en España, Francia e Italia, Iustel, 2018, pág. 391.

${ }^{13}$ LEGRAND, M., Partenariats Public Privé, Financement des biens culturels et surplus social, 2005 (Université Paris I Panthéon Sorbonne UFR de Sciences Économiques DEA mention Décisions publiques, institutions et organisations), p. 45.

${ }^{14}$ VASSILAKOU, A., MANIATIS, A., «PPP in French Law and Practice», $5^{\text {th }}$ Annual EuroMed Conference of the EuroMed Academy of Business, pp. 1621-1622.

15 EVANGELATOU, K., MANIATIS, A., MANOLIADIS, O., «PPP and Public Works Law in France», $6^{\text {th }}$ Annual EuroMed Conference of the EuroMed Academy of Business, p. 906.
} 
los visitantes. Por ejemplo, la versión del software de la emisión de billetes era instable, particularmente lenta e insatisfactoria, aumentando la cola. Es importante clarificar que la operación se normalizó en pocos meses, sin que el organismo público del museo haga uso de cualquiera otra forma de contratación pública, apoyándose en sus propios recursos humanos. Es notable que ese organismo mencionaba en su informe anual de 2008 que la actividad relativa a la Informática en materia del sistema de billetes debería externalizarse mediante un contrato de $\mathrm{CPP}^{16}$.

Ese fracaso ha sido atribuido a tres causas diversas ${ }^{17}$. En primer lugar, la razón obvia consistía en la incapacidad de gestión de las herramientas tecnológicas nuevas. En segundo lugar, existía también una decisión del Ministerio competente, de retrasar la ejecución del contrato. En tercer lugar, la adquisición del contratista, es decir de la empresa «Unilog», por la compañía «Logica» causó una atmósfera de incertidumbre a sus empleados, de los cuales algunos decidieron dejar la empresa en el momento crucial. Vista esta motivación, la «MAPPP» ha señalado que este caso fortalece su posición, según la cual la autoridad de adjudicación debe interferir lo menos posible a las obligaciones contractuales, con la excepción de disfunción.

La conclusión del acercamiento al fiasco en el museo de Versalles es que el patrimonio debe aproximarse con mucho cuidado. Por lo general, el modelo de CPP ha sido usado particularmente en Francia. Sin embargo, este país no ha sido exente de problemas relativos a la aceptación del modelo, desde 2014, a causa de un fracaso relativo a un hospital ${ }^{18}$. Resulta una confirmación de la política tradicional francesa de intervención estatal en la economía ("'dirigisme")' 19 .

\section{LA CUESTIÓN DEL DERECHO HIPOTÉTICO A LA SERENIDAD}

La felicidad se transformó en un derecho fundamental civil de mayor importancia, en la Declaración de Independencia de los Estados Unidos, de 4 de julio de 1776. En este texto revolucionario estuvo explícitamente reconocida la tríada de los derechos humanos a la vida, la libertad y la persecución de la felicidad, pero después la Constitución de los Estados Unidos omitió el derecho relativo a la felicidad. En 1789 se adoptó en Francia la declaración de los derechos del hombre y del ciudadano. Este primer texto de la Revolución francesa, en el ámbito constitucional, hace referencia al mantenimiento de la Constitución y «a la felicidad de todos» como fines de las reclamaciones de los ciudadanos. Si los textos constitucionales posteriores han ignorado el derecho a la persecución de la felicidad, es notable que esta declaración, a la cual se refiere el Preámbulo de la Constitución vigente, tiene una vigencia

\footnotetext{
${ }^{16}$ KALOGIROU, S., MANIATIS, A., «Heritage PPP Law and Management», $6^{\text {th }}$ Annual EuroMed Conference of the EuroMed Academy of Business, p. 1202.

${ }^{17}$ MANIATIS, A., VASSILAKOU, A., «French Public - Private Partnerships (PPPs) law and culture», JARR B Economics, Business, Social Sciences and Education, XVI (1) 2017, p. 23.

${ }^{18}$ BRACONNIER, S., «Le futur régime des partenariats public-privé: rupture et clarifications», RDP 3/2015, pp. 595-603.

${ }^{19}$ MANIATIS, A., GKOGKAKI, M., «A New Public Management Approach to PPP Norms», $9^{\text {th }}$ Annual Conference of the EuroMed Academy of Business, p. 1228.
} 
constitucional en el ordenamiento francés. De sobra, una parte de la doctrina por los últimos años ha procedido al reconocimiento de la naturaleza constitucional de este derecho, en el derecho comparado ${ }^{20}$. Al contrario, otra parte de la doctrina y de la jurisprudencia no pueden reconocer un derecho relativo a la felicidad ${ }^{21}$. En la medida en que exista un derecho (genérico) a la persecución de la felicidad o, al menos, a la serenidad, podría deducirse también el derecho (especial) de cada persona a vacaciones serenas $^{22}$. En este mismo contexto, correspondería al Estado a través de varias herramientas, como la CPP, garantizar la serenidad de los hombres, si no una esfera polimórfica (asociada a una gran variedad de derechos, no solamente de los nuevos y de los cuasi nuevos) para que los individuos puedan dentro de la cual perseguir la felicidad.

El derecho a la serenidad constituye un nuevo derecho hipotético, el cual aún no ha sido suficientemente reconocido, pero la legislación relativa a la contratación pública o a la contratación relativa a las obras técnicas sería útil en la materia. Por ejemplo, éste es el caso de la directiva 2014/61/UE «relativa a medidas para reducir el coste del despliegue de las redes de comunicaciones electrónicas de alta velocidad», es decir en la materia precitada de la CPP en Grecia. El texto europeo alienta a las empresas a utilizar la infraestructura física existente para conseguir una economía de recursos por lo que se refiere a los trabajos que muestran un coste significativo ${ }^{23}$. Antes de la adopción de esta normativa, algunos Estados miembros de la Unión europea ya habían adoptado medidas destinadas a reducir los costes de despliegue de la banda ancha pero estas prácticas seguían siendo escasas y dispersas ${ }^{24}$. Por eso, según la directiva, "los Estados miembros garantizarán que todo operador de red tenga derecho a negociar acuerdos relativos a la coordinación de las obres civiles con las empresas que suministren o estén autorizadas para suministrar redes de comunicaciones electrónicas con vistas al despliegue de elementos de las redes de comunicaciones electrónicas de alta velocidad"25. De sobra, las empresas precitadas tienen el derecho a solicitar a todo operador de red que realice directa o indirectamente obras civiles total o parcialmente financiadas con recursos públicos (por ejemplo, a través de CPP) la coordinación de sus obras civiles ${ }^{26}$. Tienen también el derecho a someter la cuestión al organismo nacional de resolución de controversias competente, cuando no se consiga un acuerdo sobre la coordinación de las obras civiles en el plazo de un mes a partir de la fecha de recepción de la solicitud de negociación, mientras el operador de red es dotado con el mismo derecho ${ }^{27}$.

Es notable que esta coordinación de las obras civiles no es plausible únicamente por razones económicas dado que ella puede «minimizar las molestias para la zona

\footnotetext{
${ }^{20}$ GEMMA, G., «Esiste un diritto costituzionale alla felicità?», AFDSUDC, 12, 2008, pp. 519-531.

${ }^{21}$ FERRO, G., «Nuove frontiere della tutela del turista-consumatore nello spazio giuridico europeo e antiche diatribe domestiche: riflettendo sul c.d. "'danno da vacanza rovinata"', Forum di Quaderni Costituzionali, 27 giugno 2014, n. 36.

22 MANIATIS, A., «The Right to Pursuit of Happiness and Italian Tourism Law», Tourism Development Journal, Vol. 15 (1), 2017, p. 53.

${ }^{23}$ Considerando 7 y 24 de la directiva.

${ }^{24}$ Considerando 10 de la directiva.

${ }^{25}$ Apartado 1 del artículo 5.

${ }^{26}$ Apartado 2 del artículo 5.

27 Apartado 3 del artículo 5.
} 
afectada por el despliegue de nuevas redes de comunicaciones electrónicas" ${ }^{28}$. De esta formulación del preámbulo de la directiva, no reiterada en el texto principal, resulta el derecho de cada habitante a la serenidad, amenazado por operaciones técnicas para la instalación de varias redes, incluidas las de comunicaciones electrónicas.

\section{A MODO DE CONCLUSIONES}

La CPP y los nuevos derechos constituyen innovaciones de la época corriente de la cuarta generación de derechos fundamentales. Ella ha promovido de manera más o menos importante en el derecho comparado varios derechos de esta categoría, sea del contratista sea de otras personas.

Por un lado, en el marco de la CPP han emergido algunos derechos originales, relativos a la fase preliminar o aquella de la ejecución de la contratación; éste es el caso de los derechos del contratista a seleccionar a su supervisor y a ofrecerle una remuneración suplementaria según la experiencia del derecho europeo comparado. No sería normal rechazar de manera absoluta la innovación de estos derechos, los cuales podrían considerarse como privilegios particulares (no previstos por una ley formal). Este elemento moderno del marco normativo de la contratación pública no solamente ejemplifica la particularidad del régimen de la CPP frente al derecho común de la contratación pública sino en principio está ratificado por la práctica, dado que no existen problemas (al menos obvios) relativos al ejercicio de las funciones de los supervisores. Sin embargo, sería posible reconsiderar el modo de selección del contratista dado que el sistema usado, llamado «on-off», no es exento de críticas.

Por otro lado, la CPP es asociada a los derechos fundamentales del público, aun en la fase de ejecución de las obras técnicas. Por ejemplo, ella, como la contratación por lo general, debería respetar el derecho a la serenidad de los habitantes de regiones en las cuales algunas redes de infraestructuras técnicas deberían instalarse. De sobra, sería oportuno que se transforme en un mecanismo estatal de promoción sistemática, si no prioritaria, de los derechos de cuarta generación, pero también de los cuasi nuevos derechos, como los derechos al medio ambiente y al desarrollo sostenible. Esta política no se refiere sólo a los ciudadanos sino a otras personas, como los turistas extranjeros, y a conjuntos sociales enteros, como la humanidad ${ }^{29}$. Por ejemplo, se trata de la protección y la promoción de los monumentos consistentes en patrimonio mundial (sea natural sea cultural) y del derecho universal a la participación a la Sociedad de la Información.

$* * *$

La normativa sobre la CPP constituye un derecho específico, dotado con nuevos derechos relativos ya al período de ejecución de las obras técnicas, en las cuales la CPP focaliza.

\footnotetext{
${ }^{28}$ Considerando 24 de la directiva.

29 MANIATIS, A., «PPP and the constitutional right to the environment», Constitutionalism.gr, 20.11.2015.
} 


\section{BIBLIOGRAFÍA}

BRACONNIER, S., «Le futur régime des partenariats public-privé: rupture et clarifications», RDP 3/2015.

EVANGELATOU, K., MANIATIS, A., MANOLIADIS, O., «PPP and Public Works

Law in France», $6^{\text {th }}$ Annual EuroMed Conference of the EuroMed Academy of Business.

FABI, F., LOIERO, R., PROFITI, F. S. E., Il Partenariato Pubblico - Privato nell' ordinamento giuridico nazionale, comunitario ed internazionale, Dike Giuridica Editrice, 2015.

FERRO, G., «Nuove frontiere della tutela del turista - consumatore nello spazio giuridico europeo e antiche diatribe domestiche: riflettendo sul c.d. ' danno da vacanza rovinata"'», Forum di Quaderni Costituzionali, 27 giugno 2014.

FRAILE BALBÍN, P., « ¿Tenemos derecho a la Felicidad?», Expansión, 2017.

GEMMA, G., «Esiste un diritto costituzionale alla felicità?», AFDSUDC, 12, 2008

KALOGIROU, S., MANIATIS, A., «Heritage PPP Law and Management», $6^{\text {th }}$ Annual EuroMed Conference of the EuroMed Academy of Business.

LEGRAND, M., Partenariats Public Privé, Financement des biens culturels et surplus social, 2005 (Université Paris I Panthéon Sorbonne UFR de Sciences Économiques DEA mention Décisions publiques, institutions et organisations).

MANIATIS, A., EVANGELATOU K., MANOLIADIS, O., «An assessment of the French Public - Private Partnership Law», e-JST, 11 (4), 2016.

MANIATIS, A., GKOGKAKI, M., «A New Public Management Approach to PPP Norms», $9^{\text {th }}$ Annual Conference of the EuroMed Academy of Business.

MANIATIS, A., «Derecho hidráulico y contratación pública», Observatorio Contratación Pública, 05.03.2018.

MANIATIS, A., El Derecho al Agua y la Contratación Pública. Estudio de Derecho Público, Editorial Académica Española, 2018.

MANIATIS, A., VASSILAKOU, A., «French Public - Private Partnerships (PPPs) law

and culture», JARR B Economics, Business, Social Sciences and Education, XVI (1) 2017.

MANIATIS, A., «PPP and the constitutional right to the environment», Constitutionalism.gr 20.11.2015.

MANIATIS, A., «The concession nature of Public - Private Partnership», e-JST, 11 (3),

2016.

MANIATIS, A., «The Right to Pursuit of Happiness and Italian Tourism Law», Tourism Development Journal, Vol. 15 (1), 2017.

MORENO MOLINA, J. A. «El ámbito objetivo de aplicación de la ley de contratos del sector público», Documentación Administrativa núm. 274-275, 2006. 
MORENO MOLINA, J. A., PLEITE GUADAMILLAS, F., La nueva ley de Contratos del Sector Público. Estudio sistemático, La Ley, Madrid 2008.

PARENTE, A., Principios de derecho europeo de energía, Aranzadi Thomson Reuters 2010.

RAMÍREZ MARTÍNEZ, S., «Ley de Asociaciones Público-Privadas», Energía a debate, 2012.

TORNOS MAS, J. (Coord.), El servicio de suministro de agua en España, Francia e Italia, Iustel, 2018,

VAINOPOULOS, R., MERCIER, S., Le Tourisme, Le Cavalier Bleu Éditions, 2009.

VASSILAKOU, A, MANIATIS, A., «PPP in French Law and Practice», $5^{\text {th }}$ Annual EuroMed Conference of the EuroMed Academy of Business. 